\title{
Use of holmium laser for OHVIRA syndrome treatment
}

\author{
Grzegorz Kudelaa ${ }^{1}$, Agnieszka Bialka ${ }^{2}$, Tomasz Koszutski ${ }^{1}$, Agnieszka Drosdzol-Cop ${ }^{2}$ \\ ${ }^{1}$ Department of Paediatric Surgery and Urology; John Paul II Upper Silesian Child Health Centre, \\ Medical University of Silesia, Katowice, Poland \\ ${ }^{2}$ Department of Pregnancy Pathology, Medical University of Silesia, Katowice, Poland
}

\section{Introduction}

Herlyn-Werner-Wunderlich syndrome is a rare complex of structural abnormalities of the female urogenital tract. That comprises an obstructed hemivagina in a didelphic uterus with homolateral renal agenesis/ anomaly. Hence, clinical entity are better described by the term OHVIRA — obstructed hemivagina, ipsilateral renal agenesis/anomaly (dysplasia, duplication or crossed fused ectopia) with a uterine anomaly (Fig. 1). According to researches, 36\% of Müllerian anomalies occurs with other structural abnormalities and most of these are renal anomalies.

In literature, the age of OHVIRA syndrome diagnosis is ranged from 10-29 years, on average 14 years old and is most commonly encountered 1 to 2 years after menarche. $87 \%$ of patients with OHVIRA syndrome after successful surgery will still have successful pregnancy. Therefore, it is sensible to use minimally invasive surgery in cases of OHVIRA syndrome.

\section{Case report}

A 13-year-old girl was referred to our department for evaluation of right-lower quadrant pain during menstruation, suggesting appendicitis. The patient experienced regular cycles since menarche (3 months ago). She reported dysmenorrhea, with right lower abdomen pain. Her medical history was significant for a congenitally absent left kidney as well as right adnexal cyst that was surgically removed at age 3 years. There was severe tenderness in right iliac fossa. Otherwise, her general physical and systemic examination was unremarkable with normal secondary sexual characteristics. Local genital examination revealed normal labia major and minor. Ultrasound of the patient's pelvis showed a $10 \mathrm{~cm} \times 3.3 \mathrm{~cm} \times 3 \mathrm{~cm}$ fluid space with internal septation and with spaces of high echogenicity. There was

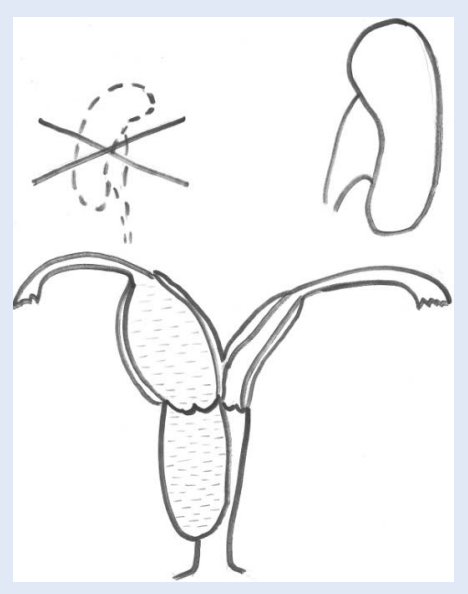

Figure 1. Schematic representation of OHVIRA syndrome

free fluid in pouch of douglas (POD). Transabdominal sonography demonstrated an absent left kidney. We proceeded with diagnostic laparoscopy under general anesthesia which revealed uterus didelphys and enlargement of the right

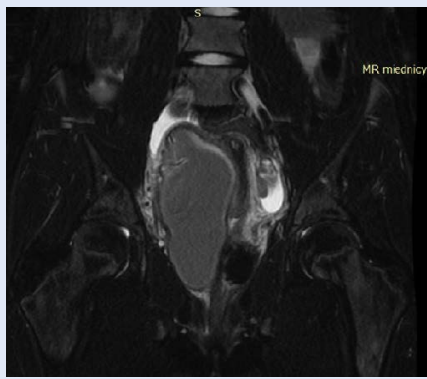

Figure 2. Magnetic resonance image showing uterus didelphys in OHVIRE syndrome uterus. A subsequent magnetic resonance imaging scan showed uterus didelphys with obstructed right hemivagina and ipsilateral renal agenesis (Fig. 2). The patient was diagnosed with OHVIRA syndrome. After adequate preparation of the patient, a pediatric cystoscope was used to performed bladder examination. Ureteral orifice was not found on the right side. Vaginoscopy revealed left vagina with lateral wall bulge formed from the right vagina. Vaginal septum was completely incised by holmium laser (Ho:YAG) extending to the cervical ostiums while hymen was not injured. Accumulated old menstrual blood was evacuated. The patient's pain was reduced. During the following months, there was still abdominal pain, but less severe, and ultrasonography showed no evidence of blood accumulation in the reproductive system. At the 9-month follow-up the patient was totally symptom-free. 


\section{Conclusion}

Due to the rarity and diversification of renal anomaly, a successful diagnosis and treatment of OHVIRA syndrome is challenging for surgeons. Procedure of removal of the septum could be performed in adolescent girls in a minimally invasive way, without damaging the hymen, using a paediatric cystoscope and holmium laser. 